Canadian Science Publishing

Journal of Unmanned Vehicle Systems Revue des systèmes de véhicules télécommandés

\title{
Mission-Based Citizen Views on UAV Usage and Privacy: An Affective Perspective
}

\begin{tabular}{|r|l|}
\hline Journal: & Journal of Unmanned Vehicle Systems \\
\hline Manuscript ID & juvs-2015-0031.R1 \\
\hline Manuscript Type: & Article \\
\hline Date Submitted by the Author: & $05-$ Feb-2016 \\
\hline Complete List of Authors: & $\begin{array}{l}\text { Winter, Scott; Florida Institute of Technology, Aeronautics } \\
\text { Rice, Stephen; Florida Institute of Technology, Aeronautics } \\
\text { Tamilselvan, Gajapriya; Florida Institute of Technology, Aeronautics } \\
\text { Tokarski, Russell; Florida Institute of Technology, Aeronautics }\end{array}$ \\
\hline Keyword: & Unmanned Aerial Vehicles, Privacy, Affect, Citizen Perceptions \\
\hline & \multicolumn{2}{l}{} \\
\hline
\end{tabular}


Running Head: UAV EMOTIONS

Mission-Based Citizen Views on UAV Usage and Privacy: An Affective Perspective

Scott R. Winter, Stephen Rice, Gajapriya Tamilselvan, and Russell Tokarski

Florida Institute of Technology

Word Count: 5205

Direct correspondence to:

Dr. Scott R. Winter

College of Aeronautics

Florida Institute of Technology

150 West University Blvd.

Melbourne, FL 32901

scott.winter@me.com

218-269-9376 


\begin{abstract}
As more uses for UAVs appear, operators, and government are struggling to identify how to incorporate these devices into the airspace system. The perceptions of citizens may play a role in whether they are accepted or rejected by society since concerns over privacy have been a major issue expressed by citizens. The purpose of these studies was to examine two UAV missions that may raise privacy concerns. In one condition, the UAV was continuously operated, in the other, the UAV was sent up for a specific mission at a time. The data suggests that citizens' concerns for privacy were less in the condition where the UAV was only used for a specific mission than when it was operated continuously. Affect measures showed that emotions play a significant role in citizen's response. Through six unique and universal facial expressions, study three identified that disgust and fear were two main emotions that mediated the relationship between the usage of the UAV and concerns over privacy. The findings of these studies may have practical implications for both operators (private and military) and regulators in the government as both struggle to decide how successful implementation of UAVs into the airspace system should occur.
\end{abstract}

Keywords: Unmanned Aerial Vehicles, Privacy, Citizens Perceptions, Affect 
Unmanned Aerial Vehicles (UAV) are increasing in capabilities. UAVs can be used for objectives such as photography, filming, agricultural applications, search and rescue, military and police resources, and for commercial operations, such as package delivery. However, as UAVs increases within the United States, there is concern over their infringement of the public's right to privacy, according to the U.S. Government Accountability Office (2016). On February 15, 2015, the Federal Aviation Administration (FAA) proposed new rules to govern small (less than 55 pounds) UAVs for use by private operators (FAA 2015). Privacy concerns were not included since they were outside the scope of this proposed rule (Kaminski 2015). The same day that the FAA released their proposed rules, President Obama also issued a presidential memorandum on balancing economic development of UAVs while also protecting civil rights and liberties (Kaminski 2015). While some States have enacted their own laws on privacy against UAV, it is unclear if these laws would stand due to possible First Amendment constitutional rights (the right in the United States that protects freedom of speech) (Kaminski 2015). As UAVs continue to integrate into the United States National Airspace System (NAS), citizen and public privacy concerns will need to be addressed. The purpose of these studies was to examine citizen perceptions on privacy based upon the mission of the UAV, and the duration it was flown: continuously or on an as needed basis. These studies sought to determine if affect measures mediate these citizen perceptions (indicating that responses were the result of the affective rather than the cognitive domain), and through the six universal faces of emotion, specifically identify, which emotions explained any mediation. In the following section, the authors provide a review of literature on privacy, UAVs, and affect.

\section{Literature on Citizen Privacy}


Review of the current literature on unmanned aerial vehicles (UAV) and citizen privacy starts with the definition of privacy as it relates to UAVs. Webster (1964) defines privacy as "the quality or condition of being private; withdrawal from public view or company; seclusion" (p. 1159), and “freedom from unauthorized intrusion" (Merriam-Webster 2015 para. 2). Villasenor's (2013) evaluated UAVs and their effects on privacy and the fourth amendment of the United States Constitution (the right in the United States that persons shall be secure in their homes and property and restricts unauthorized searches). Several law cases were decided well before the civil use of modern UAVs to identify what privacy comprises in the court of law. He divided UAV use into three categories: private, commercial and governmental. While government use of UAVs is undoubtedly regulated by the fourth amendment, private and commercial users are protected by the first amendment (Villasenor 2013). Governmental agencies usage of UAVs may not violate the privacy afforded a person's house, papers, and effects as set out in the fourth amendment. Villasenor highlights the multiple perspectives surrounding UAVs integration and the law. It will be up to the courts to decide what uses of a UAV can be deemed as violating a person's privacy, whether civil, commercial, or private.

Anderson (2014) studied privacy as a value in the principles of Privacy by Design concept. The concept of Privacy by Design, from a systems engineering perspective, is that privacy should be taken into account throughout the design of the product and includes seven statements: proactive not reactive; preventative not remedial, privacy as the default setting, privacy embedded into design, full functionality - positive-sum, not zero-sum, end-to-end security - full life-cycle protection, visibility and transparency - keep it open, and respect for user privacy - keep it user centric (Cavoukian, Taylor, \& Abrams 2010). Anderson concluded that the value of UAV privacy had to be met in a pluralistic manner dependent on the types of 
technologies used in the UAV. She suggests that the technology (such as video cameras) utilized in the UAV could in many cases conflict with a person's privacy, and the capabilities that this technology afforded law enforcement agencies would then lose its faith or trust in the agency. Because of this, she concludes that UAV technology selection and the application should be reviewed using the principles of Privacy by Design established by Cavoukin, Canada's Information and Privacy Commissioner. Utilizing this framework, law enforcement agencies would get the most use out of the UAV, while protecting the privacy of the citizens.

Choi-Fitzpatrick (2014) examined UAV use through the work of artists, journalists, and activists. He related commercial UAVs to cameras in the sense they capture current activities so they can be broadcast to the masses. The only difference is that the UAV can gain a unique, more far reaching, and often, intrusive view of one's private activities. When the camera was introduced to the masses, it experienced disdain from the public eye. Private individuals had concerns over the effect the camera would have on their privacy in public places (ChoiFitzpatrick 2014). Widespread use eventually led to societal acceptance of the camera in public areas. Getting caught on film was accepted into the popular culture and even anticipated. He also points out that to the activist and the artist, the UAV, as did the camera, has become a way to get their message out, and give a more broad view of the situation than has ever been able before (Choi-Fitzpatrick 2014).

With the UAV market expected to exceed $\$ 8.35$ billion by 2018 (US Newswire 2013), the Obama administration has ordered the National Telecommunications and Information Administration (NTIA) and the Federal Aviation Administration (FAA) to devise guidelines and rules for the use of UAVs by civil and private users. It has taken one year for this order to be executed. In August 2015, the FAA, NTIA, local and federal law enforcement, manufacturers 
and the advocates for private UAV and radio control aircraft have all met to start a dialog on rules and regulations to protect privacy but also provide the public freedom to use them as they require (Newstex 2014). The administration would like to see the FAA integrate UAVs in the national airspace system. In recent years the FAA has administered special flight permits for civil use, such as law enforcement agencies and fire departments, but the White House has made it very clear they want regulation for commercial and private users as well (Newstex 2014). During these talks, advocates have been adamant that instead of more regulation, what is needed is education on the responsible use of UAVs (Gross 2015). Their argument has revolved around their assessment that most UAV issues stem from residents becoming unnecessarily upset over UAV use because they are not educated on their utilization and capabilities. It would also follow that the UAV users should observe public privacy rules and observe the responsible use of UAVs. Following these guidelines, advocates suggest, will result in a seamless integration of UAVs into the national airspace system (Newstex 2014). From these articles, many issues related to UAV privacy depend on perception, mission, and the operator which will be experimentally examined within the current research studies.

\section{Unmanned Aerial Vehicles}

Unmanned Aerial Vehicles (UAV) usage has been expanding in recent years (Villasenor 2013). The uses of UAVs perhaps seems unlimited with some examples being traffic monitoring, police assistance, wildfire surveillance, search and rescue operations, military operations, package delivery, agricultural missions, and perhaps in the future, even passenger and cargo transportation. UAVs also come in many sizes from small toy like devices that may weigh less than a few pounds to large vehicles that resemble the size and structure of a large commercial airliner. With such a large variance in their size and capabilities, challenges are exposed not only 
to their usage, but also their safe integration into the national airspace system. UAVs also come with varying levels of technology (Villasenor 2013).

Some UAVs are restricted to line-of-sight limits, which mean they have to be in continuous sight and signal range of the operator, while others can be controlled or even programmed to operator autonomously on a preset or predetermine flight path. Some may or may not be equipped with video cameras that could record or even provide a live feedback stream to the operator. With this increased usage come concerns. Who can and should operate these aircraft? What training is necessary for these pilots? How will privacy and constitutional amendment rights be protected while UAVs are in operation? As this technology influxes society, regulating agencies will have to work quickly to successfully implement policies and procedures to countenance for their use (FAA 2015).

\section{Affect}

Research on affect and its role in evaluative processes has intensified (Bodenhausen 1993; Bower 1991; Clore, Schwarz \& Conway 1994; Zanjonc 1998). This research is emphasized on considering the influence of affect or emotions in the decision-making process. The roles of affect in any decision processes are distinct and demands meticulous research (Peters, Vastjfall, Garling, \& Slovic 2006). Many studies propose that emotions play a vital role in making decisions rapidly (Frijda 1986; Levenson 1994; Oatley \& Johnson - Laird 1996). Some studies suggest that emotions, which direct attention, memory, and judgment can influence decisions more than cognitive thought processes (Johnson -Laird \& Oatley 1992; Lazarus 1991; Schwarz 1990; Simon 1967; Tooby \& Cosmides 1990).

Earlier research has made it clear that affect and cognition are separate components (Trafimow \& Sheeran 1998, 2004; Trafimow et al. 2004), and emotions are an intrinsic part in 
executing evaluative judgments (Clore, Schwarz \& Conway, 1994; Schwarz 1990; Schwarz \& Clore 19831988 1996). There are situations highlighted where emotions play an integral role to form personal opinions. Alice (2001) claimed that positive affect is an efficient tool which enhances problem solving and decision-making. This research produces evidence that positive affect facilitates social interaction; advances to the certain qualities like helping, generosity and interpersonal understanding. Nowadays, there is ample research, from which positive affect influences thinking, and there is an absolute need to consider the situation that surrounds the decision making process.

The impacts of affect can be studied with the major functions it plays in judgment and decision making processes (Peters, Vastjfall, Garling, \& Slovic 2006). First, affect can act as information, where the decision maker just thinks at the instant of judgment to make their choice and ask themselves, "How do I feel about this?” (Schwarz \& Clore 2003). These actions may guide the judgment or decision process (Slovic et al. 2002) or it can be viewed as outcomes of prior experiences or thoughts, which catalyze picking choices on decision making processes (Damasio 1994). Second, affect facilitates exercising analogy with various decision options or information in decision-making. Affect constantly succeeds to involve as a two-step process. First, the major focus is placed on the extent or type of affective feelings (e.g. weak vs. strong affect or anger vs. fear). However, this process is superseded by new information (because of affective feelings rather than the initial feelings themselves), which ease the judgment and decision making process. Lastly, affect acts as a motivator of information processing and behavior.

Strong emotional responses have resulted in affective process, and therefore not from cognitive process. These responses can influence information processing and judgment (Zajonc 
1980), and there is evidence that affect completes mental models in situations where cognitive ability rise above rational ability (Lee $\&$ See 2004). Previous research was conducted by Alhakami and Slovic (2004), where the affect heuristic was studied. They figured out that affect is the quicker and frequent way to see a person's emotional response, whether good or bad unconsciously. Heuristics offer an optimal solution to any impossible task, as mental shortcuts that ease the decision making process, although, there is no guarantees that the correct conclusion is made with the mental shortcuts. Also, Finucane, Alhakami, Slovic, and Johnson (2000) and Loewenstein, Weber, Hsee, and Welch (2001) explored that the Affect Heuristic is inversely related to time pressure and emotional responses, respectively.

Damasio (1994) presented an effective way to explain the role of affect in decision making as the Somatic Marker Hypothesis. This research suggests that the thought process is mainly facilitated by images, which promote perceptual and symbolic representations. These images render positive (happiness, surprise, energetic) and negative (anger, disgust, fear, sadness) feelings, which influence somatic or bodily states directly or indirectly. This hypothesis ascertains to increase the accuracy and efficiency of decision-making process. The result applies to the current studies because of the impact of affect on information processing, and it is feasible to review the person's perceptions (by means of images presented) on the operation of Unmanned Aerial Vehicles (UAVs) and on their view towards UAV privacy around them.

\section{Six Universal Facial Expressions}

Research completed by Ekman and Friesen (1971) sought to determine if there was a universal relationship between facial muscular patterns and emotions. From their studies, they found six unique facial expressions that could be recognized by all individuals. The six expressions are: happiness, sadness, anger, surprise, disgust, and fear. A copy of the facial 
expressions is shown in Figure 1. In their research, participants were given a scenario, and then asked to select the facial expression that best matched that scenario. These researchers completed a comprehensive examination of subjects from varied populations: Westernized adults and subjects unexposed to any Western ideals before. Ekman and Friesen found that the majority of participants from both groups recognized these expressions regardless of cultures, western influence, and countries of origin.

This method was also used in a previous study by Cremer and Rice (in press) and Rice and Winter (2015). In that study, the researchers examined citizen's willingness to fly on an aircraft that had a human pilot or one that used only an autopilot (no human pilot on-board). The six universal facial expressions were used to find that citizens preferred the human pilot and the emotions of anger, fear, and happiness mediated the relationship between the condition of the pilot and citizen's willingness to fly. In the current experiments, this study uses these universal faces as an unbiased way to collect data on which emotions were influencing participants in their perceptions toward UAV use and privacy.

\section{Current Studies}

The purpose of the current studies was to examine citizen perceptions toward privacy based on using UAVs to complete a police mission. As UAV usage has increased, public concern over the right to privacy and $4^{\text {th }}$ Amendment rights has been expressed. The current studies sought to determine how citizens would view two hypothetical scenarios of UAV usage: 1) the UAV was deployed 24 hours a day and 2) the UAV was only deployed on a specific mission basis. The studies proposed the following hypotheses:

$\mathrm{H}_{1}$ : Citizens would have more privacy concerns when the UAV was operated 24 hours a day compared to only being operated by police for a specific mission. 
$\mathrm{H}_{2}$ : Affect would mediate the relationship between UAV mission usage and privacy; that is, the relationship between the UAV mission usage and privacy is only present when affect is included in the model.

$\mathrm{H}_{3}$ : The ratings of the six universal facial expressions would change for the various UAV usage and differential mediation effects (some emotions will have a different mediating effect than others) would be present between UAV usage and privacy ratings.

The current research project was completed as three studies. First, a study was completed to determine if there would be a difference in privacy concerns between the UAV usages. Study 2 included a general affect measure to find if affect would mediate the relationship between UAV usage and privacy, and finally, Study 3, utilized the six universal faces to identify which emotions were acting as mediators between UAV usage and privacy.

\section{Study 1 - Methods}

Participants. Two hundred (85 females) participants from the United States were recruited via a convenience sample using Amazon's ${ }^{\circledR}$ Mechanical Turk ${ }^{\circledR}$ (MTurk). MTurk provides participants who complete human intelligence tasks for monetary compensation. Prior research shows that data from MTurk is as reliable as normal laboratory data (Buhrmester, Kwang, \& Gosling 2011; Germine, et al. 2012). The mean age was $32.02(S D=9.17)$.

Materials and Stimuli. Participants first signed an electronic consent form, and then were given instructions about the study. Following this, they were presented with a hypothetical scenario about the use of unmanned aerial vehicles (UAV) for police activities. Specifically, in one condition, they were told, "Imagine a situation where your local police department announces plans to use unmanned aerial vehicles (UAVS) to patrol the skies of your neighborhood 24/7 (day and night) every day of the year in order to assist with police activities. These UAVs would fly at 
various altitudes and provide aerial coverage with video feedback of the entire neighborhood at all times." In another condition, they were told, "Imagine a situation where your local police department announces plans to use unmanned aerial vehicles (UAVS) to assist with police activities. These UAVs would fly at various altitudes and provide aerial coverage with video feedback of the affected streets and/or areas and then return to the police station at the completion of the mission."

Following this, participants were asked to complete a validated UAV privacy scale (Mehta, Rice, Winter \& Oyman 2015). This scale can be found in Appendix A. Lastly, participants were asked basic demographics questions, debriefed, and remunerated for their time.

\section{Study 1 - Results}

First, the data from the UAV privacy scale was subjected to a Cronbach's Alpha test to ensure reliability. The scores were .96 and .97 for the two conditions, showing high reliability in the data. Next, the two conditions were compared using a two-tailed independent samples t-test, $t(198)=2.52, p=.01, d=.36$, indicating that the scenario where the police would use $24 / 7$ coverage $(M=1.11, S D=.82)$ resulted in more privacy concerns compared to the scenario where the police only used specific activity coverage $(M=.79, S D=1.00)$.

\section{Study 2 - Methods}

Participants. One hundred and ninety (78 females) participants from the United States were recruited via a convenience sample using Amazon's ${ }^{\circledR}$ Mechanical Turk ${ }^{\circledR}$ (MTurk). The mean age was $33.47(S D=11.12)$.

Materials and Stimuli. Study 2 was identical to Study 1 with the following exception. After being presented with the scenarios, and before responding to the UAV privacy scale, participants 
were asked to respond to an affect scale validated by Rice and Winter (2015). This scale can be found in Appendix B.

\section{Study 2 - Results}

First, the data from the UAV privacy scale was subjected to a Cronbach's Alpha test to ensure reliability. The scores were .97 and .96 for the two conditions, showing high reliability in the data. Next, the data from the affect scale was subjected to a Cronbach's Alpha test to ensure reliability. The scores were .97 and .98 for the two conditions, indicating high reliability in the data.

For the affect scores, the two conditions were compared using a two-tailed independent samples t-test, $t(188)=2.49, p=.01, d=.36$, indicating that the scenario where the police would use $24 / 7$ coverage $(M=-0.83, S D=1.04)$ resulted more negative affect compared to the scenario where the police only used specific activity coverage $(M=-0.46, S D=1.00)$. For the privacy scores, the two conditions were compared using a two-tailed independent samples t-test, $t(188)=$ $2.96, p=.003, d=.43$, indicating that the scenario where the police would use $24 / 7$ coverage $(M$ $=.98, S D=.86)$ resulted in more privacy concerns compared to the scenario where the police only used specific activity coverage $(M=.57, S D=1.03)$.

Mediation Analyses. The standardized regression coefficient between UAV usage and affect was statistically significant as was the relationship between affect and privacy. The standardized indirect effect was $(.37)(-0.74)=.27$. The significance of this indirect effect was tested using bootstrapping procedures (Hayes, 2013) with 10,000 bootstrapped samples. The bootstrapped $95 \%$ confidence interval ranged from -0.50 to -0.06 . Thus, the indirect effect was statistically significant, and there was mediation. Therefore, the relationship between the UAV 
mission usage and privacy scores either does not exist, or is significantly weaker, when affect is excluded from the model as a mediator.

\section{Study 3 - Methods}

Participants. One hundred and ninety-four (64 females) participants from the United States were recruited via a convenience sample using Amazon’s ${ }^{\circledR}$ Mechanical Turk ${ }^{\circledR}$ (MTurk). The mean age was $31.52(S D=10.23)$.

Materials and Stimuli. Study 3 was identical to Study 2 with the following exception. Instead of using the general affect scale used in Study 2, we instead used a more specific affect scale (Rice \& Winter in press) that allowed participants to rate how they felt about the target individual based on images (see Figure 1) of the six universal emotions (Ekman \& Friesen 1971). Participants were shown each image in random order and asked, "Based on the scenario above, how strongly do you feel like the image shown?" Participants manipulated a slider with their computer mouse. The slider had scale ends of "I do not feel this way at all" to "I extremely feel this way". The slider scored the responses on a numerical scale from $0-100$; however, participants were not aware of this.

\section{Study 3 - Results}

For the affect scores, the data were subjected to a mixed-design two-way ANOVA, with Condition (between-participants) and Type of Emotion (within-participants) as the two factors. There was a main effect of Condition, $F(1,192)=8.40, p=.004$, partial-eta squared $=.04$, and a main effect of Type of Emotion, $F(5,960)=18.08, p<.001$, partial-eta squared $=.09$. These main effects are qualified by a significant interaction between the two factors, $F(5,960)=3.96, p$ $=.001$, partial-eta squared $=.02$, showing that the difference between the two conditions was moderated by the type of emotion. These data are presented in Figure 2. 
Next, the data from the UAV privacy scale was subjected to a Cronbach's Alpha test to ensure reliability. The scores were .96 and .97 for the two conditions, showing high reliability in the data. The two conditions were compared using a two-tailed independent samples t-test, $t(192)$ $=2.73, p=.007, d=.39$, indicating that the scenario where the police would use $24 / 7$ coverage $(M=1.09, S D=.87)$ resulted in more privacy concerns compared to the scenario where the police only used specific activity coverage $(M=.71, S D=1.09)$.

Mediation Analyses. While there was a significant difference for Type of Emotion, the researchers wanted to find which of the six emotions, if any, were mediators. Using the Preacher and Hayes (2008) bootstrapping method of multiple mediation analysis, we found statistically significant indirect effects for Disgust (CI ranges: -.15, -.004), and Fear (-.17, -.02), with effect sizes of .04, and .07 , respectively. Thus, mediation occurred for these two emotions.

\section{General Discussion}

The purpose of these studies was to examine citizen perceptions on UAVs for a police mission. The length of UAV deployment was manipulated to research how citizen's perceptions toward their privacy would be influenced. Affect measures were collected to understand if participant's answers resulted from a cognitive or emotional response after reading the scenarios. Finally, through the six universal faces, the studies attempted to find which emotions were the ones influencing the relationship between UAV usage and privacy.

The first hypothesis predicted that there would be a difference in citizen's perceived level of privacy based on whether the UAV was continually present ( 24 hours a day) or if it was only launched on specific missions. The data across the three studies support this hypothesis. Participants were much more concerned over their privacy when the UAV was airborne 24 hours a day than when it was used for a specific mission and returned to base. As UAV operations and 
usages continue to grow, citizens are concerned over their privacy. Similar to the concerns over privacy when the video camera was produced (Choi-Fitzpatrick 2014), citizens may be experiencing similar reactions to UAV presence. When the UAV is deployed on a certain mission, in these studies a fairly generic example was provided, it is possible that citizens see the advantage of the UAV usage out weighing their concerns for privacy. News outlets have recently shown numerous examples where UAVs have assisted after natural disasters to video flood zones, etc. This perceived gain in what can be provided by the UAV perhaps help explain why citizens have less privacy concerns than when deployed full time. While the UAV is airborne 24 hours a day, the mission may be less clear to the citizen since there is no perceived benefit to its usage, but perhaps a disadvantage, as the citizen does not see the point of its operation or perceives that it could just be collecting images of normal daily activity, which threatens the right to privacy.

The concepts of Cavoukian, Taylor, and Abrams (2010) highlight Privacy by Design. These seven principles may assist UAV operators in addressing some of the privacy concerns held by the public. Specifically, being proactive not reactive, privacy embedded into design, visibility and transparency, and respect for privacy. Through being proactive, the citizens can be informed of the advantages that are provided through UAV usage. As people become more aware of the many uses, cost savings, and benefits offered by UAVs, it is possible they may become more accepting of the technology. Also, showing how privacy issues may be embedded into the design of the UAV and/or policies that support privacy could ease citizen privacy concerns. Just as other forms of evidence are only made admissible in court proceedings, policies created for UAV use may enhance the understanding of UAV operations. Finally, if UAVs are visible and transparent and there are clear protocols to respect privacy, it is viable that citizens 
would become more accepting of the advantages and uses of UAVs for the greater good of the community.

The second hypothesis predicted that affect would mediate the relationship between UAV usage and privacy. The data from the studies supports this hypothesis. Affect has been shown capable of influencing thought processes (Schwarz \& Clore 2003; Slovic et al. 2002). When faced with having to determine one's privacy concerns about UAV usage, it is plausible that emotions may influence or explain at least part of this response. With UAV usage occurring more frequently, it is possible that citizens do not fully understand the advantages or procedures associated with UAV operations. It is also possible that government and private operators have not clearly provided this information to the public. Either way, when this gap in understanding occurs, it is possible that emotional factors could heavily influence one's reactions and perceptions toward UAV usage (Lee \& See 2004).

The final hypothesis predicted that through the six universal faces, it might be possible to find which emotions mediate the relationship between UAV usage and privacy. The data suggest that disgust and fear were the two emotions that citizen's felt in their concerns over privacy. Through identifying these two emotions, it is possible to gain a better understanding of citizen's privacy concerns. With disgust, citizens may feel that the usage of the UAVs is revulsive or arouses unpleasant or offensive feelings. The thought of a UAV being capable of gathering private information would certainly cause feelings of disgust in most people, especially if they felt that the UAV had no policies or procedures to limit what it could or could not examine. Fear is also a very plausible and unpleasant emotional response. If citizens view UAVs as dangerous, this could trigger the emotion of fear or if the UAV usage is viewed as a threat to persons and/or property. 


\section{Practical Implications}

There are several practical implications from the current studies, and these findings may interest UAV operators, the private sector, and perhaps government regulators. First, as UAV's enter the national airspace system, concerns over citizen's privacy will need to be addressed. While there appear to be advantages in the missions that can be completed via UAV's, this economic benefit for the agencies will have to be balanced against citizen concerns over privacy. Of particular interest in the current studies was the finding that disgust and fear were to two mediating emotions expressed by the participants. It may be possible that since UAV's are new, people have little experience with them operating around, such as they would with perhaps something like a helicopter. Other possible explanations could be UAV's have been used in military operations and entertainment portrayals where UAVs are spying or destroying objects. This may associate a stigma with them, and UAV's can take on many shapes and sizes. While more research is needed to investigation the reason for this affective response, it is possible that more familiarity and education about UAV's and the missions these devices are capable of may play a role in changing citizen's perceptions of their usage and their concerns over personal privacy. Since disgust and fear were the two emotions shown that mediate the relationship between UAV usage and privacy, it is possible that citizens felt threatened, believed UAVs were dangerous, and had a sense that the UAV violated their rights to privacy. Knowing it appears to be these emotions that are influencing citizen's views to UAVs and privacy, it is possible that greater information on UAV usage, privacy policies, procedures for safe operations, etc. may help citizen's gain a better understanding toward the possible benefits offered by UAV usage.

\section{Limitations and Recommendations for Future Research}


The current studies were bound by certain limitations. While MTurk has been shown to provide valid and reliable data, the findings from the studies cannot be generalized beyond those in the American population that complete online human intelligence tasks. Further research can expand on the population used for these studies to see if the findings replicate. These studies only looked at one mission UAV's can complete: a police mission. Privacy concerns may vary based the mission the UAV is completing. Additional research is needed to determine how the mission affect privacy concerns in individuals. Finally, who is operating the UAV may also affect privacy concerns. Manipulation of this variable may show insights into citizen privacy and perhaps an interaction between the mission and operator for citizen privacy concerns.

\section{Conclusions}

The purpose of these studies was to examine how citizen's views on privacy would be affected when a UAV was used on a police mission. In one scenario, the UAV was continuously operated, while in the other condition, the UAV was only operated on one specific mission at a time. Citizen's preferred the scenario where the UAV was only operated on a single mission than when the UAV was continuously deployed. Additionally, affect was shown to mediate the relationship between the UAV operation and citizen's perceived levels of privacy. Disgust and fear were the two main emotions that appear to be mediating the relationship. As the proliferation of UAV uses increases, concerns over citizen's right and level of privacy will need to be addressed. The data from these studies suggests that citizens were experiencing an emotional response when considering their views toward privacy and presence of the UAV. The findings of these studies may benefit operators and the government when working to identify how to successfully incorporate the usage of UAV's into the national airspace system and dayto-day operations and missions. 
UAV Emotions 20

\section{References}

Alhakami, A. S., \& Slovic, P. (1994). A psychological study of the inverse relationship between perceived risk and perceived benefit. Risk Analysis, 14, 1085-1096.

Anderson, S. (2014). Privacy By Design: An Assessment of Law Enforcement Drones (Master's thesis, Georgetown University). Retrieved from http://search.proquest.com.portal.lib.fit.edu/docview/1531333351?pqorigsite $=$ summon $\&$ accountid $=27313$

Bodenhausen, G. V. (1993). Emotions, arousal, and stereotypic judgments: A heuristic model of affect and stereotyping. In D .M. Mackie \& D. L. Hamilton (Eds.), Affect, cognition, and stereotyping: Interactive processes in group perception (pp. 13-37). San Diego, CA: Academic Press.

Bower, G. H. (1991). Mood congruity of social judgment. In J. Forgas (Ed.), Emotion and social judgment (pp. 31-54). Oxford, UK: Pergamon.

Buhrmester, M., Kwang, T., \& Gosling, S. D. (2011). Amazon’s Mechanical Turk: A new source of inexpensive, yet high-quality data? Perspectives on Psychological Science, 6(3), 3-5.

Cavoukian, A., Taylor, S., \& Abrams, M. E. (2010). Privacy by design: Essential for organizational accountability and strong business practices. Identity in the Information Society, 3, 405-413.

Choi-Fitzpatrick, A. (2014). Drone for good: Technological innovations, social movements, and the state. Journal of International Affairs, 68(1), 19-XI.

Clore, G. L., Schwarz, N., \& Conway, M. (1994). Cognitive causes and consequences of emotion. In R. S. Wyer, Jr. \& T. K. Srull (Eds.), Handbook of social cognition (2nd ed., pp. 323-417). Hillsdale, NJ: Erlbaum. 
Cremer, I. \& Rice, S. (in press). Which emotions mediate the relationship between type of water recycling projects and likelihood of using green airports? International Journal of Sustainable Aviation.

Damasio, A.R. (1994). Decartes' error: Emotion, reason and the human brain, New York: Avon.

Ekman, P. \& Friesen, W.V. (1971). Constants across cultures in the face and emotion. Journal of Personality and Social Psychology, 17(2), 124-129.

Federal Aviation Administration. (2015). Press release: DOT and FAA propose new rules for small unmanned aircraft systems. Retrieved from: https://www.faa.gov/news/press_releases/news_story.cfm?newsId=18295

Finucane, M. L., Alhakami, A., Slovic,P., \& Johnson, S.M. (2000). The affect heuristic in judgments of risks and benefits. Journal of Behavioral Decision Making, 13, 1-17.

Frijda, N. H. (1986). The emotions. Cambridge, UK: Cambridge University Press. Bulletin, 117, 39-66.

Germine, L., Nakayama, K., Duchaine, B.C., Chabris, C.F., Chatterjee, G., \& Wilmer, J.B. (2012) Is the web as good as the lab? Comparable performance from web and lab in cognitive/perceptual experiments. Psychonomic Bulletin \& Review, 19(5), 847-857.

Gross, G. (2015). U.S. to begin talks on drone privacy standards. Computerworld. Advance online publication. Retrieved from www.computerworld.com.au

Isen, A., M. (2001). An influence of positive affect on decision making in complex situation: Theoretical issues with practical implications. Journal of Citizen Psychology, 11(2), 7585.

Johnson-Laird, P. N., \& Oatley, K. (1992). Basic emotions, rationality, and folk theory. 
UAV Emotions 22

Cognition and Emotion, 6, 201-223.

Kaminski, M. E. (2015, February 25). The rules of the sky. [web blog post on Slate: Future Tense]. Retrieved from:

http://www.slate.com/articles/technology/future_tense/2015/02/faa_small_commercial_dr

one_rules_don_t_adequately_address_privacy_concerns.single.html

Lazarus, R.S. (1991) . Emotion and adaptation. New York: Oxford University Press.

Lee, J. D., \& See, A. K. (2004). Trust in automation: Designing for appropriate reliance. Human Factors, 46, 50-80.

Mehta, R., Rice, S., Winter, S. R., \& Oyman, K. (2015). Citizen perceptions of privacy toward the usage of unmanned aerial systems: A valid and reliable instrument. 8th Annual Human Factors and Applied Psychology Student Conference, Daytona Beach, FL.

Merriam-Webster. (2015). Online dictionary. Retrieved from: http://www.merriamwebster.com/dictionary/privacy

Oatley, K., \& Johnson-Laird, P. N. (1996). The communicative theory of emotions: Empirical tests, mental models, and implications for social interaction. In L. L. Martin \& A. Tesser (Eds.), Striving and feeling: Interactions among goals, affect, and self-regulation. Hillsdale, NJ: Erlbaum.

Peters, E., Vastjfall, D, Garling, T, \& Slovic, P. (2006). Affect and decision making: A “hot” topic. Journal of Behavioral Decision Making, 19, 79-85.

Rice, S. \& Winter. S. (2015). A quick affect scale: Providing evidence for validity and reliability. 10th International Conference on Interdisciplinary Social Sciences. 
Rice, S. \& Winter, S.R. (2015). Which emotions mediate the relationship between type of pilot configuration and willingness to fly? Journal of Aviation Psychology and Applied Human Factors 5(2), 83-92.

Schwarz, N. \& Clore, G.L. (1983). Mood, misattribution and judgments of well-being: Informative and directive functions of affective states. Journal of Personality and Social Psychology, 45, 513- 523.

Schwarz, N. \& Clore, G. (1988). How do I feel about it? The information function of affective states. In K. Fiedler \& J.P. Forgas (Eds.), Affect, cognition, and social behavior: New evidence and integrative attempts (pp. 44-63). Toronto: C.J. Hogrefe.

Schwarz, N., \& Clore, G. (1996). Feelings and phenomenal experiences. In E. T. Higgins \& A.W. Kruglanski (Eds.), Social psychology: Handbook of basic principles (pp. 433-465). New York: Guilford Press.

Schwarz, N., \& Clore, G. L. (2003). Mood as information: 20 years later. Psychological Inquiry, 14(3), 296-303.

Simon, H. A. (1967). Motivational and emotional controls of cognition. Psychological Review, 74, 29-39.

Slovic, P., Finucane, M. L., Peters, E., \& MacGregor, D.G. (2002). The affect heuristic. In T. Gilovich, D. Griffin, \& D. Kahneman (Eds), Heuristics and biases: The psychology of intuitive judgment. (pp. 397-420).

Trafimow, D., \& Sheeran, P. (1998). Some tests of the distinction between cognitive and affective beliefs. Journal of Experimental Social Psychology, 34, 378-397.

Trafimow, D., \& Sheeran, P. (2004). A theory about the translation of cognition into affect 
and behavior. In G. Maio \& G. Haddock (Eds.), Contemporary perspectives in the psychology of attitudes: The Cardiff Symposium (pp. 57-76). London: Psychology Press.

Trafimow, D., Sheeran, P., Lombardo, B., Finlay, K. A., Brown, J., \& Armitage, C.J. (2004). Affective and cognitive control of persons and behaviors. British Journal of Social Psychology, 43, 207-224.

Tooby, J., \& Cosmides, L. (1990). The past explains the present: Emotional adaptations and the structure of ancestral environments. Ethology and Sociobiology, 11, 375-424.

Unmanned aerial vehicle (UAV) market worth $\$ 8351.1$ million by 2018. (2013, June 13). U.S. Newswire. Retrieved from: http://search.proquest.com/docview/1367084935?accountid=27313

United States Government Accountability Office (2016). Unmanned Aerial Systems (Drones). Retrieved from: http://www.gao.gov/key_issues/unmanned_aerial_systems/issue_summary

Villasenor, J. (2013). Observations from above: Unmanned aircraft systems and privacy. Harvard Journal of Law and Public Policy, 36, 457-517.

Webster's. (1964). Webster's new world dictionary of the American language. New York, USA: The World Publishing Company.

White House working on privacy guidelines for citizen drones [Newstex comment]. (2014). Retrieved from Newstex: http://search.proquest.com/docview/1618234653?accountid=27313

Zajonc, R. (1998). Emotions. In D. Gilbert, S. Fiske, \& G. Lindzey (Eds.), The handbook of social psychology (Vol. 1, pp. 591-632). New York: Oxford University Press. 

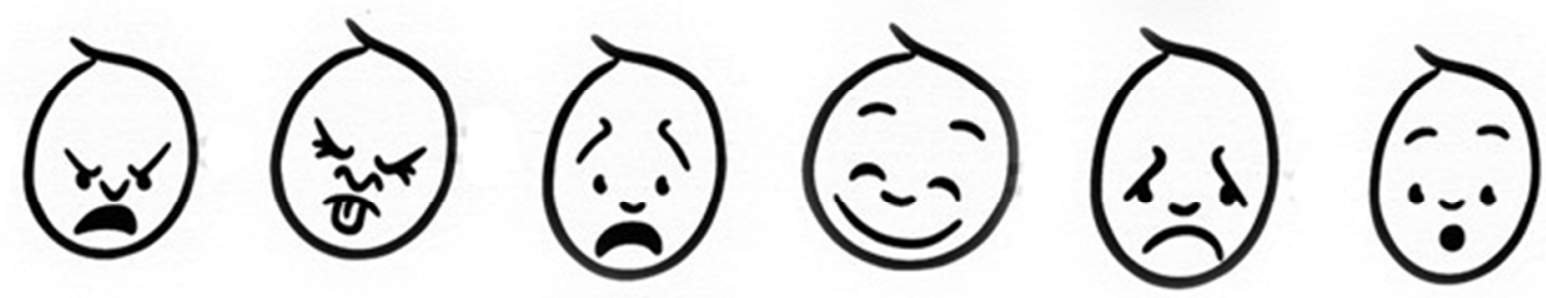

Figure 1. Six emotions from Ekman and Friesen's (1971) work are represented here with images. These images were re-validated in a separate pilot study. They represent anger, disgust, fear, happiness, sadness and surprise. 


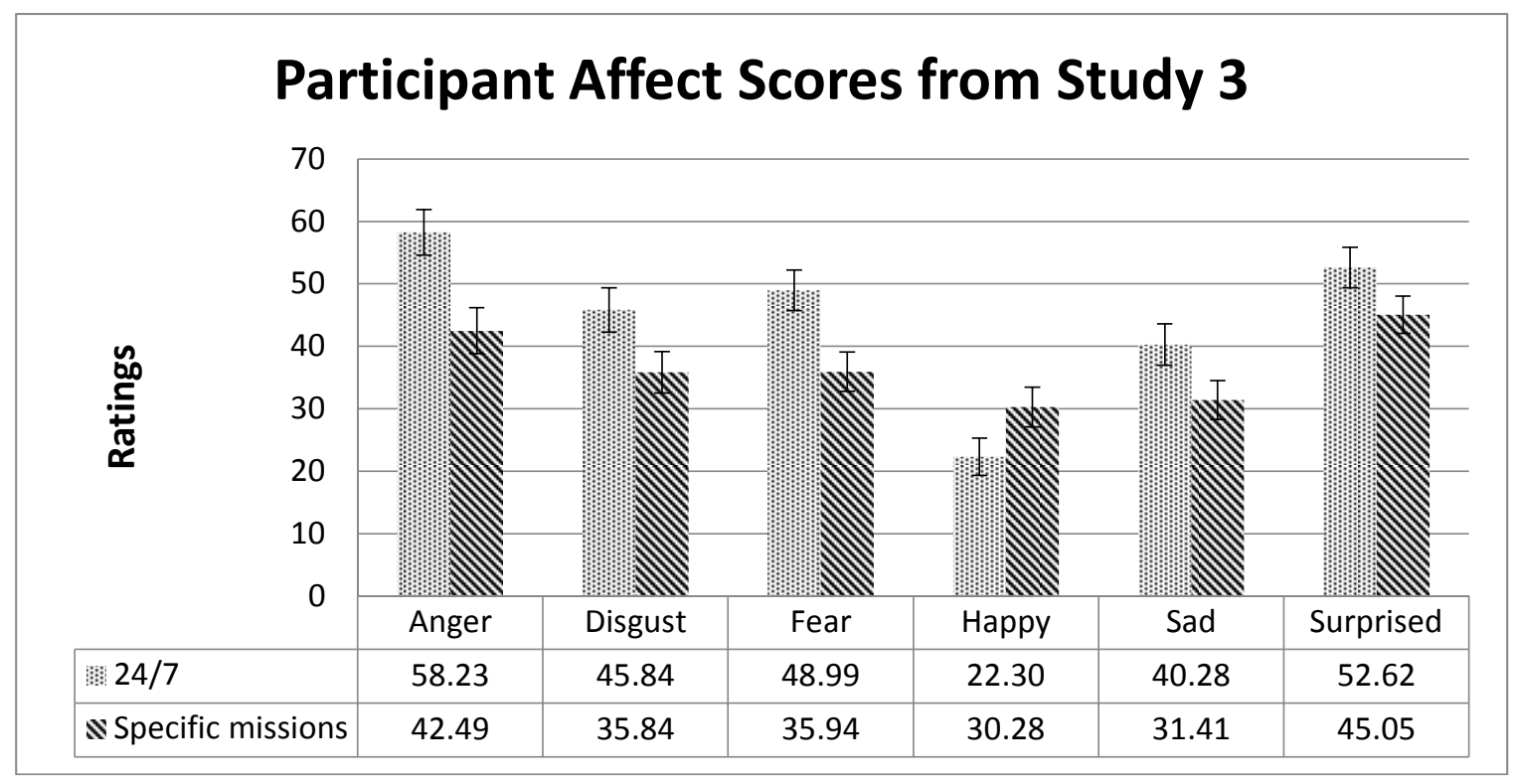

Figure 2. Affect data for participants from Study 3 and Ekman and Friesen's (1971) six emotions. SE bars are included. 
Appendix A - UAV Privacy Scale (Mehta et al., 2015).

Please respond how strongly you agree or disagree with the following statements:

1. In this situation, I believe that my privacy could be violated by the presence of this UAV.

Strongly Disagree Disagree Neutral Agree Strongly Agree

2. I believe that the owner/operator of this UAV could have access to too much private information about me in this situation.

Strongly Disagree Disagree Neutral Agree Strongly Agree

3. I believe that my control over my own privacy would be lessened in this situation.

Strongly Disagree Disagree Neutral Agree Strongly Agree

4. I would not be comfortable with how much information this UAV could gather about me in this situation.

Strongly Disagree Disagree Neutral Agree Strongly Agree

5. In this situation, the amount of private information that this UAV could gather about me makes me feel uncomfortable.
Strongly Disagree
Disagree Neutral
Agree Strongly Agree

6. This situation makes me concerned about how my privacy could be violated by the presence of this UAV.

Strongly Disagree Disagree Neutral Agree Strongly Agree

7. This situation generates privacy concerns with regards to the presence of this UAV.

Strongly Disagree Disagree Neutral Agree Strongly Agree

8. In this situation, I feel my loss of privacy is greater than what can be gained through usage of this UAV.

Strongly Disagree Disagree Neutral Agree Strongly Agree

9. In this situation, the presence of this UAV poses a threat to my privacy.

Strongly Disagree Disagree Neutral Agree Strongly Agree 
Appendix B - Affect Scale (Rice \& Winter, 2015)

Please respond how strongly you agree or disagree with the following statements:

1. I feel good about this.

Strongly Disagree Disagree Neutral Agree Strongly Agree

2. I feel positive about this.
Strongly Disagree
Disagree
Neutral
Agree
Strongly Agree

3. I feel favorable about this.

Strongly Disagree Disagree Neutral Agree Strongly Agree

4. I feel cheerful about this.

Strongly Disagree Disagree Neutral Agree Strongly Agree

5. I feel happy about this.

Strongly Disagree Disagree Neutral Agree Strongly Agree

6. I feel enthusiastic about this.

Strongly Disagree Disagree Neutral Agree Strongly Agree

7. I feel delighted about this.

Strongly Disagree Disagree Neutral Agree Strongly Agree 